\title{
Connectivity and Coverage in Hybrid Wireless Sensor Networks using Dynamic Random Geometric Graph Model
}

\author{
Jasmine Norman, \\ Vellore Institute of Technology, Vellore - 14
}

\begin{abstract}
Random Geometric Graphs have been a very influential and well-studied model of large networks, such as sensor networks, where the network nodes are represented by the vertices of the RGG, and the direct connectivity between nodes is represented by the edges. This assumes homogeneous wireless nodes with uniform transmission ranges. In real life, there exist heterogeneous wireless networks in which devices have dramatically different capabilities. The connectivity of a WSN is related to the positions of nodes, and those positions are heavily affected by the method of sensor deployment. As sensors may be spread in an arbitrary manner, one of the fundamental issues in a wireless sensor network is the coverage problem. In this paper, I study connectivity and coverage in hybrid WSN based on dynamic random geometric graph.
\end{abstract}

\section{KEYWORDS}

WSN, Random Geometric Graph, Connectivity, Coverage

\section{INTRODUCTION}

The integration of different wireless access technologies combined with the huge characteristic diversity of supported services in next-generation wireless systems creates a real heterogeneous network. In contrast to a traditional static wireless sensor network which consists of a large number of small sensor nodes with low computational, storage and communication capabilities [12], such limitations no longer apply in a mobile sensor network. In real world, at a given time, there may be stationary, mobile and powerful base stations existing together in a region. Assuming all the nodes know their destination ID, when an event occurs or when requested by the base station, they try to forward the data to their base station. The topology changes continuously due to the mobility of the nodes. It will be practically impossible to directly forward the data to the base station most of the times due to the nature of radio signals. Hence the problem is to find a neighbour (hop) towards the destination. This is done repeatedly till the destination is reached. In a heterogeneous setup there may be a few base stations or powerful sink nodes in a region. 
International journal on applications of graph theory in wireless ad hoc networks and sensor networks (GRAPH-HOC) Vol.3, No.3, September 2011

The connectivity of a WSN is related to the positions of nodes, and those positions are heavily affected by the method of sensor deployment [11] . In general, there are two types of approaches to deploy sensors in a WSN: deterministic deployment, where sensors are placed exactly at preengineered positions, and the random deployment, where nodes are deployed at random positions. For the deterministic deployment, networks are carefully planned, and nodes are placed at desired positions. If specifications of nodes are known, it is not difficult to determine whether the network is connected, and if not, to add relay nodes where needed. Although deterministic deployment has many advantages, in order to reduce installation costs, it has often been proposed that large WSNs which contain very large numbers of nodes be deployed randomly. Nodes may be dispersed by a moving vehicle or artillery shell. Mobile sensors also add to the dynamic nature of the network. Therefore, sensors often have nondeterministic positions, and the analysis of connectivity of such a type of networks involves the modeling of random networks.

Connectivity and coverage problems [10] are caused by the limited communication and sensing range. To solve both problems the solution lays in how the sensors are positioned with respect to each others. Coverage problem is regarding how to ensure that each of the points in the region to be monitored is covered by the sensors. It is a unique problem, in maximizing coverage the sensors need to be placed not too close to each other so that the sensing capability of the network is fully utilized and at the same time they must not be located too far from each other to avoid the formation of coverage holes (area outside sensing range of sensors). On the other hand from connectivity point of view, the sensors need to be placed close enough so that they are within each other communication range thus connectivity is ensured.

Random graphs are typically used to represent sensor networks. The authors in $[2,8,15]$ have studied the application of random geometric graph to wireless sensor networks. Chen Avin in [2] had investigated the property of random geometric graphs that has implication for routing and topological control in sensor networks. The goal was to construct a special subgraph, the Restricted Delaunay Graph, that permits efficient routing, based only on local information. In $[1,13,18]$ the authors studied the toplogy and connectivity properties of random geometric graphs.

In this paper I analyse the connectivity and coverage properties of dynamic RGG that suits hybrid WSN.

\section{RELATED WORK}

Due to the limited transmission range, sensors that are far away from the sink deliver their data through multihop communications. Although there is active research on maximizing network lifetime or network capacity most of these work consider a sensor network under a given physical topology. Random graphs are often applied to model communication networks to highlight their randomness. Mathematically, a random graph is a graph that is generated by a stochastic process . The theory of random graphs began with Erdos and Renyi's pioneer work in the late 1950s and early 1960s, which considered a class of random graphs now called Erdos-Renyi graphs [9]. As the probability of an edge existing between each pair of nodes is equal in an Erdos-Renyi graph, this model is not well suited to WSNs, which are embedded in two (or three) dimensional space, and in which the probability of a link existing is very much higher between nodes which are geometrically close. A natural candidate for random network modeling is the class of Random Geometric Graphs [8]. With node set V , a geometric graph $\mathrm{G}=(\mathrm{V}, \mathrm{r})$ is equivalent to a graph $\mathrm{G} 1$ 
International journal on applications of graph theory in wireless ad hoc networks and sensor networks (GRAPH-HOC) Vol.3, No.3, September 2011

$=(\mathrm{V}, \mathrm{E})$, in which the vertex set $\mathrm{V}$ is embedded in a metric space, and $E=\{(\mathrm{u}, \mathrm{v}) \mid \forall \mathrm{u}, \mathrm{v} \in$ $\mathrm{V},\|\mathrm{u}-\mathrm{v}\|<\mathrm{r}\}$.

One of the most interesting questions regarding the connectivity of random WSNs concerns finding limiting regimes for which the connectivity becomes almost sure to occur. Among the most celebrated results is that of Gupta and Kumar [6,7]. This showed that for $\mathrm{n}$ nodes uniformly, independently and identically distributed (i.i.d.) in a disk of area A, with the particular radio transmission range is given by (1).

$$
\mathrm{r}(\mathrm{n})=\sqrt{A(\log n+\gamma n)) /(\pi n)}
$$

In ad-hoc and sensor networks, interference grows with increased communication radius. So, for a property of interest $\mathrm{Q}$ of random geometric graphs, one wants to find a tight upper bound on the smallest radius $r_{c o n}(n)$, that will guarantee that $Q$ holds with high probability (w.h.p.). The radius $r_{c o n}(n)$ is called the critical radius if $Q$ exhibits a sharp threshold, that is if the difference between the smallest radius for which the property holds with high probability and the largest radius for which the property holds with low probability goes to 0 as $n->\infty$. The critical radius for connectivity, $r_{c o n}$, has been of special interest, and it has been shown that if

$$
r \geq r_{c o n}=\sqrt{\frac{\log n+\gamma n}{\pi n}}
$$

then $\mathrm{G}(\mathrm{n}, \mathrm{r})$ is connected w.h.p.as $\mathrm{n}->\infty$ iff $\mathrm{n}->+\infty$ and disconnected w.h.p.iff $\mathrm{n}->-\infty$. In adhoc and sensor networks we usually require that the network is connected, so information can be exchanged across the whole network. Therefore the critical radius for connectivity, $r_{\text {con }}$, is used as a reference point.

The authors in $[8,15]$ have studied the application of random geometric graph to wireless sensor networks. In $[4,5,11]$ the authors studied the topology and connectivity properties of random geometric graphs. In [4] the authors show that the random strategy is the only way to deploy future WSN and therefore prove that the modeling with Random Geometric Graphs is the most appropriate for WSN.

The authors in [5] have studied the large connectivity of dynamic random geometric graph. In [9] I proposed to find the nearest base station for efficient routing in heterogeneous networks. A random geometric graph with radius $r$ as the transmission range of the node is constructed. If there is a base station or a dominant node exists within the graph, the node is said to be covered. In this context the essential properties of optimized routing namely, a one dominant RGG and vertex cover are identified. Also a new graph model HRGG is proposed to suit heterogeneous sensor networks.

When sensor nodes are deployed in a non-static environment, the connection topology will change over time. If nodes in a network are mobile and the network is not supported by stationary 
International journal on applications of graph theory in wireless ad hoc networks and sensor networks (GRAPH-HOC) Vol.3, No.3, September 2011

relays and routers, the connectivity can vary. Additionally, the changes will vary quickly over time. Mobile scenarios are e.g. the monitoring of a herd of wild animals, tagging of objects of animals swimming in rivers or seas, ice-clods or any similar effect from natural movement.

Since sensors may be spread in an arbitrary manner, one of the fundamental issues in a wireless sensor network is the coverage problem $[3,10,14]$. In general, this reflects how well an area is monitored or tracked by sensors. In the literature, this problem has been formulated in various ways. Localized exposure-based coverage and location discovery algorithms are in literature. On the other hand, some works are targeted at particular applications, but the central idea is still related to the coverage issue. For example, sensors' on-duty time should be properly scheduled to conserve energy. Since sensors are arbitrarily distributed, if some nodes share the common sensing region and task, then we can turn off some of them to conserve energy and thus extend the lifetime of the network. This is feasible if turning off some nodes still provide the same "coverage" (i.e., the provided coverage is not affected).

In this paper the issue of connectivity and coverage are discussed based on dynamic RGG.

\section{CONNECTIVITY}

Dominating-set-based routing is based on the concept of dominating set in graph theory . A subset of the vertices of a graph is a dominating set if every vertex not in the subset is adjacent to at least one vertex in the subset. The main idea of this approach is to reduce the routing and searching process to a subgraph induced from the dominating set. Moreover, the dominating set should be connected for the ease of the routing process within the induced graph consisting of dominating nodes only. Vertices in a dominating set are called gateway hosts while vertices that are outside a dominating set are called non-gateway hosts. The main advantage of connected dominating-set-based routing is that it simplifies the routing process to that in a smaller subnetwork generated from the connected dominating set. This means that only gateway hosts need to keep routing information. As long as changes in network topology do not affect this sub network there is no need to re-calculate routing tables.

In [ 9] a new graph model HRGG based on RGG is suggested for hybrid WSN. The model works based on a metric NSM (Nearest Sink node). For connectivity, the following condition should hold.

$$
\mathrm{D}(\mathrm{n}, \mathrm{Bn})<=\operatorname{Tr}(\mathrm{n})
$$

Let $\mathrm{n}$ be the node that wishes to send data. As per the model $\mathrm{n}$ has to find the nearest base station or a powerful node to forward the data. Let $\mathrm{Bn}$ be a powerful node / any base station. If the distance between the node $\mathrm{n}$ and $\mathrm{Bn}$ is less than are equal to the transmission range of the node $\mathrm{n}$, then the graph is connected. There are a few scenarios to exploit after a link is established.

1. The node and the base station move in the opposite directions.

2. The node and the base station move towards each other.

3. The node is static and the BS moves away.

4. Both the BS and the node are static. 
International journal on applications of graph theory in wireless ad hoc networks and sensor networks (GRAPH-HOC) Vol.3, No.3, September 2011

In cases 3 and 4 , there is less scope to analyse in the context of HRGG. In case I, as soon as the condition is violated, the link will be cut off and HRGG will be disconnected. Case 2 actually strengthens the link as both the node $\mathrm{n}$ and the base station will be in closer proximity. As in static setup, there is no possibility of having a monotonically increasing or decreasing graph as the links are determined by the position and the position is unpredictable in hybrid setup. Thus in order to have connectivity, the HRGG has to be a one dominant connected graph.

\section{COVERAGE}

The coverage problem is difficult to address in hybrid WSN. A naive solution is to find out all sub-regions divided by the sensing regions of all $\mathrm{n}$ sensors (i.e., $\mathrm{n}$ circles), and then check if each sub-region is k-covered or not. Managing all sub-regions is a difficult and computationally expensive job in geometry because there could exist as many as $\mathrm{O}(\mathrm{n} 2)$ sub-regions divided by the circles. Also, it may be difficult to calculate these sub-regions due to the mobile nature of the nodes. I present a simple scheme based on HRGG model.

When a node senses an event, all the nodes in the region are likely to detect the event. So it is enough if the node is assured of a powerful node in its own region. The node checks the route table and if there is a sink node within its vicinity, it goes off to sleep. As the base station or the powerful node also would sense the data and so it is expected to forward the data thus conserving energy of the other nodes. When there is no powerful node around, the node tries to identify a neighbour and forward the data. So coverage is possible if

1. There exists a BS within its area - At least a one dominant graph

2. There exists a node which can forward - There exists an edge between a node $\mathrm{n}$ and any other node.

Otherwise the node has to wait for a period of ' $t$ ' seconds and refresh the route table. This waiting continues till a new node moves to its region.

\section{IMPLEMENTATION}

I studied this protocol on GloMoSim, [16,17] a scalable discrete-event simulator developed by UCLA. This software provides a high fidelity simulation for wireless communication with detailed propagation, radio and MAC layers. The GloMoSim library is used for protocol development in sensor networks. The library is a scalable simulation environment for wireless network systems using the parallel discrete event simulation language PARSEC.

The best neighbour is computed based on the type of the node and its location. The sample node file used in Glomosim is given below. The fields are in the order of node no, type and the location. Type 0 is a static node, type 1 is a rechargeable mobile node and type 2 is a powerful sink node/ base station.

$00(21.5,23,0.11)$

$11(24.5,20,0.01)$

$22(19.5,19,0.12)$

$30(22.5,15,0.05)$

$40(24.5,12,0.09)$ 
International journal on applications of graph theory in wireless ad hoc networks and sensor networks

(GRAPH-HOC) Vol.3, No.3, September 2011

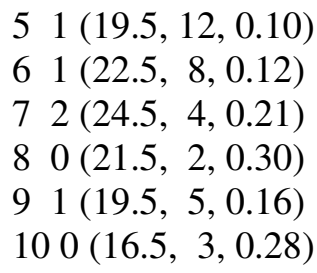

Table 1 Sample Reachability matrix

\begin{tabular}{|c|c|c|c|c|c|}
\hline 0 & 2 & 1 & 32 & 34 & 36 \\
\hline 1 & 2 & 24 & 0 & 35 & - \\
\hline 2 & 26 & 30 & 33 & 45 & 7 \\
\hline 3 & 30 & 22 & - & - & - \\
\hline 4 & 5 & - & - & - & - \\
\hline
\end{tabular}

A reachability matrix is formed as in Table 1 , representing each node and its neighbours arranged in the order of best neighbours. Thus if the node has a base station near it, it will occupy the first position. The matrix can be used for finding whether a path exists between the source and the destination at time ' $t$ '. The connectivity can be determined based on the above matrix. Also it is easier to divide the nodes into mutually exclusive sets based on the best neighbour. For example, the best neighbour of the nodes 0 and 1 is node 2 . Thus $(0,1,2)$ gets into a set. When one node transmits, the remaining nodes will go to the sleep mode thus conserving energy. The highest energy node in the set is chosen to transmit data. As the route table is built dynamically based on the mobility of the nodes, the set also changes.

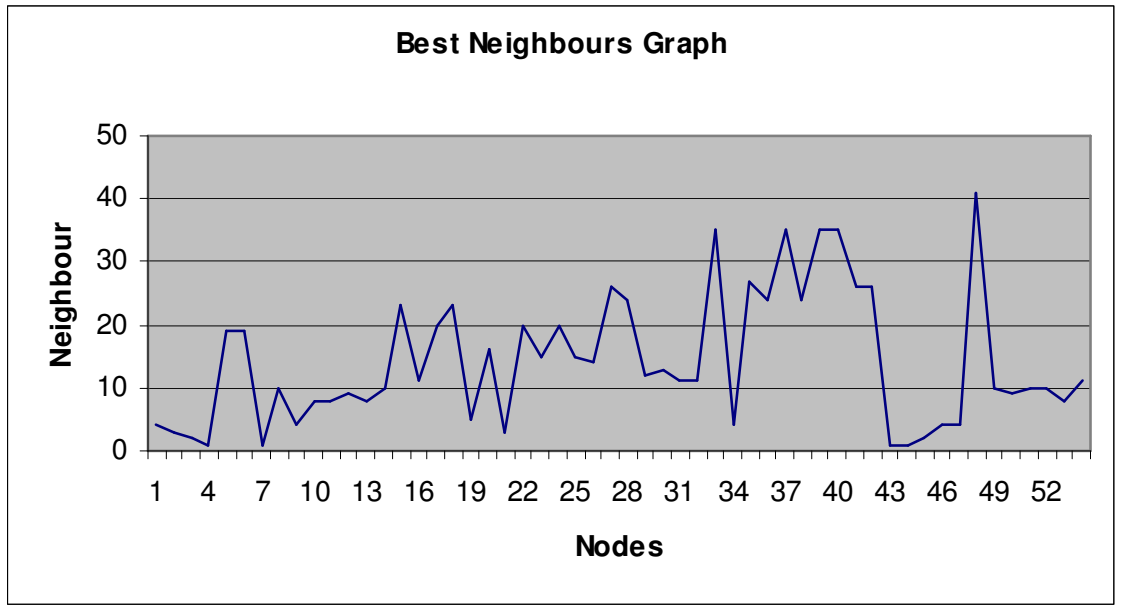

Figure 1 : Best Neighbour for Each Node 
International journal on applications of graph theory in wireless ad hoc networks and sensor networks (GRAPH-HOC) Vol.3, No.3, September 2011

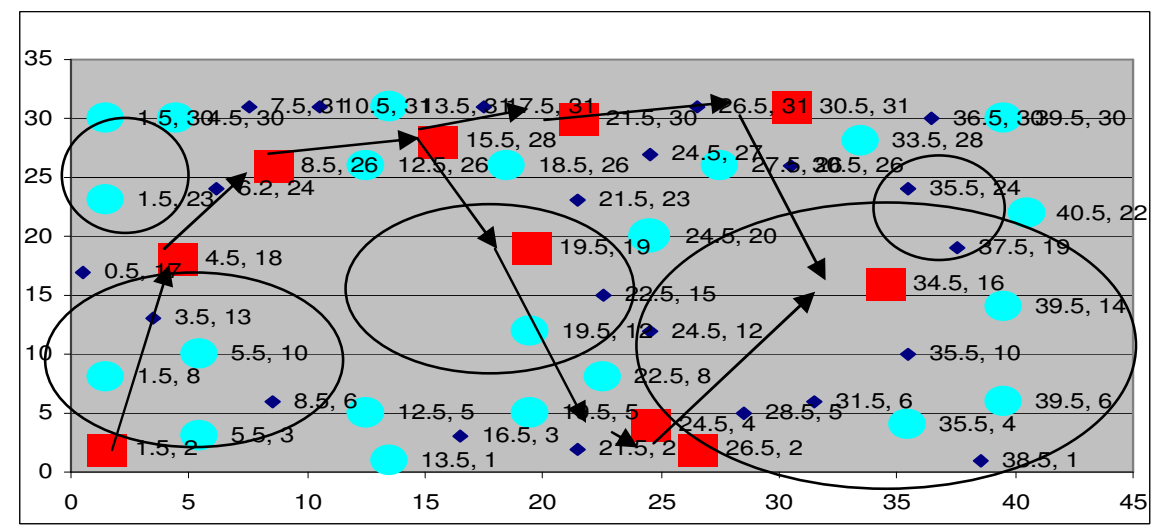

Figure 2 : Connectivity graph of the sample nodes.

Figure 2 gives the optimized connectivity as the base stations are spread apart. The stationary and the mobile sensors are spared of their energy in transmission in this particular scenario.

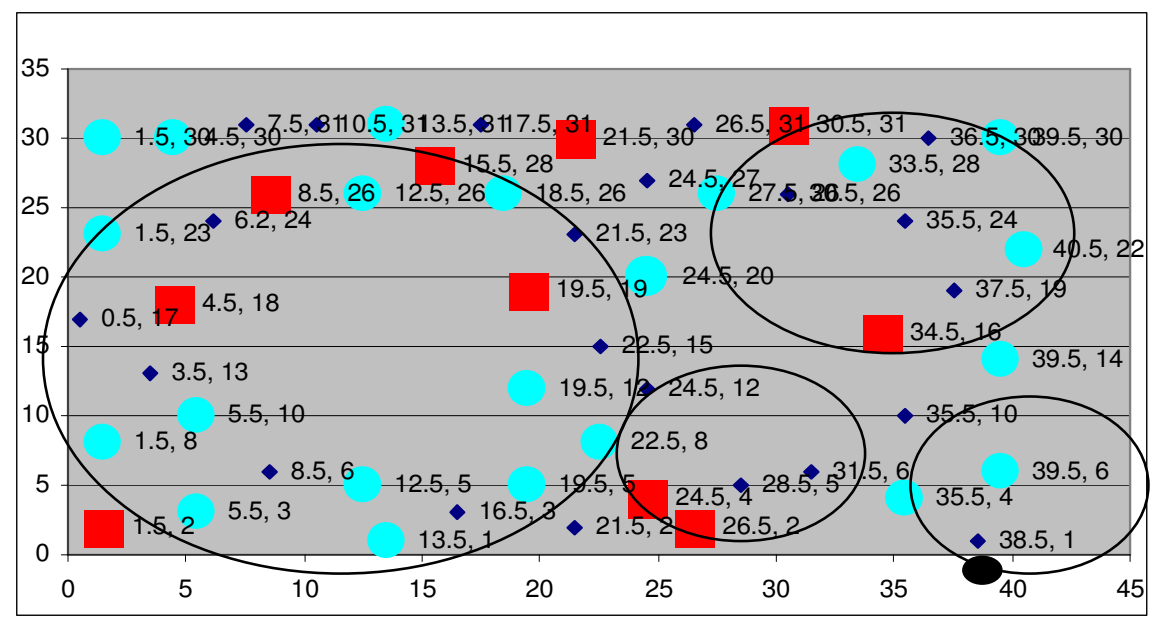

Figure 3 : Coverage graph of the sample nodes.

Figure 3 shows the mutually separated regions. Only one node in the region will forward the data. The nodes are scheduled in such a way that the highest energy node is selected first to transmit. If there happens be a base station or a powerful node all the other nodes are spared of their energy. Thus the given scheme gives maximum coverage and is energy efficient.

\section{CONCLUSION}

In this paper the connectivity and coverage properties of hybrid WSN are discussed based on dynamic RGG model. Connectivity is based on one dominant dominating set and coverage is based on transmission range and scheduling of the nodes. In this effect, in order to conserve energy of the network, it is proposed to divide areas into mutually exclusive sets based on the sink nodes present. The simulation results also prove that the given model is energy efficient and is highly feasible. 
International journal on applications of graph theory in wireless ad hoc networks and sensor networks

(GRAPH-HOC) Vol.3, No.3, September 2011

\section{REFERENCES:}

[1] Bhupendra Gupta , Srikanth K Iyer, D Manjunath , “Topological Properties Of The One Dimensional Exponential Random Geometric Graph”, Random Structures \& Algorithms , Volume 32 , Issue 2 , 2008, pp: 181-204

[2] Chen Avin, "Random Geometric Graphs: An Algorithmic Perspective" , Ph,D dissertation, University of California , Los Angeles , 2006

[3] Chi-Fu Huang, Yu-Chee Tseng, “The Coverage Problem in a Wireless Sensor Network” , WSNA'03, September 19, 2003, San Diego, California, USA.

[4] J. D'1az D. Mitsche X. P'erez-Gim'enez , “On the Connectivity of Dynamic Random Geometric Graphs, Symposium on Discrete Algorithms" , Proceedings of the nineteenth annual ACM-SIAM symposium on Discrete algorithms , 2008, pp 601-610

[5] Josep Di'az, Dieter Mitsche, and Xavier Pe'rez-Gime'nez, "Large Connectivity for Dynamic Random Geometric Graphs”, IEEE Transactions On Mobile Computing, Vol. 8, No. 6, June 2009

[6] Gupta, P.; Kumar, P.R., "Critical Power for Asymptotic Connectivity in Wireless Networks", In Stochastic Analysis, Control, Optimization and Applications: A Volume in Honor of W.H. Fleming; McEneaney, W.M., Yin, G.G., Zhang, Q., Eds.; Birkhauser Boston: Cambridge, MA, USA, 1998; 1106-1110.

[7] Gupta, P., Kumar P.R, “The Capacity of Wireless Networks”, IEEE Trans. Inform. Theory 2000, 46, 388404.

[8] Hichem Kenniche, Vlady Ravelomananana, "Random Geometric Graphs as Model of Wireless Sensor Networks", IEEE , 2010

[9] Jasmine Norman , J.Paulraj Joseph , "A New Graph Model for Heterogeneous WSN", Communications in Computer and Information Science, Springer Berlin Heidelberg, July 2011 , 187-196

[10] Ji Li , Lachlan L.H. Andrew, Chuan Heng Foh , Moshe Zukerman and Hsiao-Hwa Chen , “Connectivity, Coverage and Placement in Wireless Sensor Networks “, Sensors 2009, 9, 7664-7693

[11] Krohn, A.; Beigl, M.; Decker, C.; Riedel, T.; Zimmer, T.; Varona, D.G., "Increasing Connectivity in Wireless Sensor Network using Cooperative Transmission”, In Proceedings 3rd International Conference on Networked Sensing Systems (INSS), Chicago, IL, USA, 2006.

[12] F. L. LEWIS , “Wireless Sensor Networks - Smart Environments: Technologies, Protocols, and Applications”, ed. D.J. Cook and S.K. Das, John Wiley, 2004

[13] Ning Li , Jennifer C. Hou , “Topology Control in Heterogeneous Wireless Networks: Problems and Solutions", IEEE/ACM Transactions on Networking (TON), Volume 13, Issue 6, 2005, pp 1313 1324

[14] Nor Azlina Ab. Aziz, Kamarulzaman Ab. Aziz, and Wan Zakiah Wan Ismail , “Coverage Strategies for Wireless Sensor Networks”, World Academy of Science, Engineering and Technology 502009

[15] Penrose, M.D. "Random Geometric Graphs”, Oxford University Press: Oxford, UK, 2003. 
International journal on applications of graph theory in wireless ad hoc networks and sensor networks (GRAPH-HOC) Vol.3, No.3, September 2011

[16] M. Takai, L. Bajaj, R, Ahuja, R. Bagrodia and M. Gerla, "GloMoSim: A Scalable Network Simulation Environment”, Technical report 990027, UCLA , 1999

[17] Wireless Sensors Location Data : http://www.select.cs.cmu.edu/data/index.html

[18] Xiang-Yang Li , Wen-Zhan Song, Yu Wang, "Localized topology control for heterogeneous wireless sensor networks" , ACM Transactions on Sensor Networks (TOSN), Volume 2 , Issue 1 , 2006, pp 129 - 153 